\title{
Fungal Keratitis - Improving Diagnostics by Confocal Microscopy
}

\author{
E. Nielsen ${ }^{\mathrm{a}}$ \\ S. Heegaard ${ }^{b, c}$ \\ J.U. Prause \\ A. Ivarsen ${ }^{a}$ \\ K.L. Mortensen ${ }^{d}$ \\ J. Hjortdal ${ }^{a}$
}

${ }^{a}$ Department of Ophthalmology, Aarhus University Hospital, Aarhus, ${ }^{b}$ Eye Pathology Institute, Department of Neuroscience and Pharmacology, University of Copenhagen, Copenhagen, 'Department of Ophthalmology, Glostrup Hospital, Glostrup, and

${ }^{\mathrm{d}}$ Department of Clinical Microbiology and Infectious Diseases, Aarhus University Hospital, Skejby, Denmark

\section{Key Words}

In vivo confocal microscopy · Fungal keratitis · Diagnostics

\begin{abstract}
Purpose: Introducing a simple image grading system to support the interpretation of in vivo confocal microscopy (IVCM) images in filamentous fungal keratitis. Setting: Clinical and confocal studies took place at the Department of Ophthalmology, Aarhus University Hospital, Denmark. Histopathological analysis was performed at the Eye Pathology Institute, Department of Neuroscience and Pharmacology, University of Copenhagen, Denmark. Methods: A recent series of consecutive patients with filamentous fungal keratitis is presented to demonstrate the results from in-house IVCM. Based upon our experience with IVCM and previously published images, we composed a grading system for interpreting IVCM images of filamentous fungal keratitis. Results: A recent case series of filamentous fungal keratitis from 2011 to 2012 was examined. There were 3 male and 3 female patients. Mean age was 44.5 years (range 12-69), 6 out of 17 (35\%) cultures were positive and a total of 6/7 (86\%) IVCM scans were positive. Three different categories of IVCM results for the grading of diagnostic certainty were formed. Conclusion: IVCM is a valuable tool for diagnosing filamentous fungal keratitis. In order to improve the reliability of IVCM, we suggest implementing a simple and clinically applicable grading system for aiding the interpretation of IVCM images of filamentous fungal keratitis.

(c) 2013 S. Karger AG, Basel
\end{abstract}




\section{Introduction}

Fungal keratitis is a devastating and potentially blinding corneal disease. Fungi are a rare cause of microbial keratitis in temperate climates but a common pathogen in warm and humid tropical climates where fungal keratitis has been reported to account for $30-62 \%$ of all microbial keratitis cases [1-3]. The majority of these fungal infections are caused by filamentous species of fungi, with Fusarium solani being the most predominant $[2,4]$.

A fast and reliable diagnosis of the condition is essential to ensure an optimal outcome $[1,2]$. The gold standard method of diagnosis is still by means of culture of corneal samples on fungal growth media. Unfortunately, the filamentous fungi grow slowly which causes a delay in the diagnosis. Moreover, the sensitivity of culture is low, ranging from 52 to $68 \%$ [ $3,5]$.

Clinically, fungal keratitis is characterized as white cotton-wool-like infiltrates with satellite lesions and feathery edges; these features are not always present, however, which renders the slit-lamp examination nondiagnostic [1]. As a consequence, keratitis caused by filamentous fungi remains one of the most challenging to diagnose, often with a considerable delay. There is a need for faster and improved diagnostics.

In vivo confocal microscopy (IVCM) of the cornea has been known for some time for its clinical applicability in investigating atypical forms of keratitis. IVCM is performed in a similar manner to a slit-lamp investigation. It is noninvasive, but requires physical contact through a fluid-coupling agent; eye drops of a certain viscosity are usually used. It generates images from the cornea with a resolution of $1 \mu \mathrm{m}$ [6], which is enough to yield instant imaging of organisms that are larger than a few micrometers, like Acanthamoeba cysts and fungal hyphae. This potentially provides an instant and reliable diagnosis. Unfortunately, a clinical consensus in the interpretation of IVCM images is still lacking.

First, by presenting a recent series of consecutive patients from 2011 to 2012, we demonstrate that our results with diagnosing filamentous fungal keratitis by IVCM are comparable to recent studies [6-13]. Second, based on our experiences and the available literature, we propose a grading system for the interpretation of IVCM images in filamentous fungal keratitis, in order to improve diagnosis and clinical decision-making.

\section{Case Series}

We studied a consecutive series of 6 patients with verified fungal keratitis from 2011 to 2012. Five patients were referred to our clinic from secondary ophthalmological centers. There were 3 male and 3 female patients with a mean age of 44.5 years (range 12-69). All patients were contact-lens users (table 1). All underwent a full ophthalmological investigation and IVCM, and a corneal sample was cultured. A positive diagnosis was based on known gold-standard methods, i.e. cultures or histopathology. All patients gave informed consent prior to the investigation.

\section{Cultures}

Sterile cotton swabs were used for corneal sampling. Samples were examined using chocolate-, 5\% blood- and Sabouraud-agar plates. PCR tests for viruses were made. The Sabouraud-agar plates were incubated for at least 7 days. The referring departments examined the samples from the $5 \%$ blood- and Sabouraud-agar plates. 


\section{Histopathology}

Examinations were made on corneal buttons in 3 cases and on 1 human eye. Histological sections were analyzed using standardized paraffin techniques including hematoxylin and eosin, periodic acid-Schiff and Grocott stains.

In vivo Confocal Microscopy

A single operator (E.N.) with 4 years of experience performed all IVCM scans. We used the HRT III laser confocal microscope (Heidelberg Engineering, Heidelberg, Germany), with a Rostock corneal module and joystick (prototype, Universitätsaugenklinik, Rostock, Germany). Sterile single-use tomocaps were used for each examination. Viscotears (Alcon, Fort Worth, Tex., USA) $2 \mathrm{mg} / \mathrm{g}$ was used as a coupling agent. Scans were performed as fulldepth 'sequence' scans, both in the center and along the edges of the infiltrates. Images were analyzed and hyphae-like structures were registered.

\section{Grading System}

IVCM images of normal and diseased corneas (e.g. dystrophies and inflammatory disorders) were compared to IVCM images from filamentous fungal keratitis. We searched the literature for IVCM images of filamentous fungal keratitis. Based on these, we formed 3 different categories of results.

\section{Results}

\section{Case Series of Filamentous Fungal Keratitis}

Six out of 17 (35\%) cultures performed for fungal keratitis were positive (table 1). However, when looking at the cultures from our laboratory alone, 5 out of $8(62.5 \%)$ were positive. Mean delay from start of symptoms until verification of diagnosis was 41 days (range 28-55). IVCM images from $F$. solani cases were positive in 4/4 (100\%) cases and 1/2 scans from the Aspergillus fumigatus case was positive. Ultimately, a total of 6/7 (86\%) IVCM scans were positive.

\section{Grading System}

We formed 3 different categories of IVCM results for grading of diagnostic certainty (fig. 1). In this system, an IVCM image can be given 1 of 3 grades depending on the level of diagnostic certainty: (1) positive for fungi, (2) inconclusive or (3) negative for fungi. Representative images are seen in figure 1.

\section{Category 1: Positive for Fungi}

This category consists of two types of images. The first shows linear, highly reflective structures in a smooth, well-defined branching and interlocking pattern. The second shows isolated, well-defined, highly reflective and bifurcating branch-like elements. Typically, the latter is found in superficial layers whereas the former is found in deeper stromal layers. Naturally occurring elements that mimic either of these two types are highly unlikely (fig. 1, top row: from patients with verified fungal keratitis).

\section{Category 2: Inconclusive}

The images in category 2 show atypical, linear and highly reflective stromal structures. They are more densely distributed and more disorganized than normal stromal nerves. These elements can represent fungal hyphae, disorganized collagen bundles or nerves and, 
occasionally, they can be found in cases with bacterial keratitis. They differ from category 1 in that they lack branch-like morphology, smoothness and well-defined features (fig. 1, middle row: from patients with verified filamentous fungal keratitis).

Category 3: Negative for Fungi

Category 3 images show normally occurring, linear structures. They lack the welldefined nature and morphological features mentioned in category 1 , and they do not exist in the dense and atypical manner as do the stromal elements in category 2 . Consequently, there is no suspicion of fungal keratitis (fig. 1, bottom row: from patients without fungal keratitis).

\section{Discussion}

From our experience and demonstrated by this recent case series from 2011 to 2012, IVCM seems superior to cultures in diagnosing filamentous fungal keratitis. The sensitivity of our IVCM was $86 \%$, which is comparable to previous reports of $80-94 \%[6,7,9]$. There are now several studies of IVCM in filamentous fungal keratitis that compare the sensitivity of cultures to IVCM and find the latter to be superior [6-13]. These results in unison could warrant a fresh look at IVCM as an accepted means of diagnosing filamentous fungal keratitis.

Unfortunately, there is no clinical consensus in the area of IVCM, and as a result individual operators are forced to base the interpretation of images on their own experiences and on previously published images. This makes IVCM highly susceptible to interobserver variation and, until a consensus can be made, its clinical application will remain limited as a stand-alone tool. Hau et al. [14] elegantly demonstrated this in 2010 in the first IVCM study using masked observers. Furthermore, IVCM images of fungal hyphae vary to a certain degree, and other corneal structures such as disorganized collagen and nerves can mimic the hyphae [8].

In trying to address these issues, we have created a grading system for the interpretation of IVCM images in filamentous fungal keratitis that can be applied clinically. This is based upon our years of experience with IVCM images in cases with verified filamentous fungal keratitis as well as on previously published studies in this field [6-13, 15].

When searching the literature for published HRT-RCM IVCM images of $F$. solani hyphae, we found 3 studies. One is by Brasnu et al. [10] in 2007, with an image similar to the superficial-type hyphae demonstrated in figure 1 (top row: middle and right panels). Three studies $[6,10,15]$ published IVCM images similar to the stromal-type hyphae demonstrated in figure 1 (top row: left panel) and figure 2 (2a).

With aid from published studies and our own experiences, we created the grading system at hand, aiming to supply IVCM operators with a simple and clinically applicable tool with the potential for standardizing the interpretation of images and suggesting an appropriate clinical approach.

We recommend that category 1 IVCM results are designated as being pathognomonic for fungal keratitis. Antifungal treatment should be initiated at once, not waiting for the results of cultures (table 1). Category 2 results are inconclusive and should not warrant diagnosis of fungal keratitis in their own right, but increased awareness is advised and the findings should be supplemented with culture or histopathology. Category 3 results are regarded as negative for fungal keratitis.

Finally, another method of diagnosing filamentous fungal keratitis is by polymerase chain reaction (PCR) of fungal-specific DNA sequences. Two clinical studies that compared 
PCR analysis with cultures in patients with fungal keratitis reported that PCR demonstrated superior sensitivities of 70 and $93 \%$, but low specificities of 57 and $43 \%$, respectively [16, 17]. PCR is faster than culturing, typically taking $4-8 \mathrm{~h}$ to perform, but the extraction of falsepositives remains a challenge due to the ubiquitous nature of filamentous fungi.

\section{Conclusion}

IVCM is a valuable tool in diagnosing filamentous fungal keratitis. In order to improve the reliability of IVCM, we suggest implementing a simple and clinically applicable grading system for aiding the interpretation of IVCM images of filamentous fungal keratitis. We recommend that IVCM be performed with minimal delay in nonresponding atypical keratitis, especially when associated with the use of contact lenses.

\section{Acknowledgements}

We thank Dr. Andrey Zhivov, Universitätsaugenklinik Rostock, for useful consultation regarding the interpretation of confocal images in patient No. 1 and patient No. 5. The HRTIII-RCM unit was kindly donated by the Jørgen Bagenkop Nielsens Myopi-Fond.

\section{References}

1 Chang P HY, Chodosh J: Diagnostic and therapeutic considerations in fungal keratitis. Int Ophthalmol Clin 2011;51:33-42.

2 Thomas PA: Fungal infections of the cornea. Eye 2003;17:852-862.

3 Srinivasan M: Fungal keratitis. Curr Opin Ophthalmol 2004;15:321-327.

-4 Keay LJ, Gower EW, Iovieno A, et al: Clinical and microbiological characteristics of fungal keratitis in the United States, 2001-2007: a multicenter study. Ophthalmology 2011;118:920-926.

5 Keay L, Edwards K, Naduvilath T, et al: Microbial keratitis predisposing factors and morbidity. Ophthalmology 2006;113:109-116.

6 Kumar RL, Cruzat A, Hamrah P, et al: Current state of in vivo confocal microscopy in management of microbial keratitis. Semin Ophthalmol 2010;25:166-170.

7 Vaddavalli P, Garg P, Sharma S, et al: Role of confocal microscopy in the diagnosis of fungal and acanthamoeba keratitis. Ophthalmology 2011;118:29-35.

-8 Takezawa Y, Shiraishi A, Noda E, et al: Effectiveness of in vivo confocal microscopy in detecting filamentous fungi during clinical course of fungal keratitis. Cornea 2010;29:1346-1352.

$\$ 9$ Kanavi MR, Javadi M, Yazdani S, et al: Sensitivity and specificity of confocal scan in the diagnosis of infectious keratitis. Cornea 2007;26:782-786.

10 Brasnu E, Bourcier T, Dupas B, et al: In vivo confocal microscopy in fungal keratitis. Br J Ophthalmol 2007;91:588-591.

11 Shi W, Li S, Liu M, et al: Antifungal chemotherapy for fungal keratitis guided by in vivo confocal microscopy. Graefes Arch Clin Exp Ophtalmol 2008;246:581-586.

12 Das S, Samant M, Garg P, et al: Role of confocal microscopy in deep fungal keratitis. Cornea 2009;28:11-13.

-13 Avunduk AM, Beuerman RW, Varnell ED, et al: Confocal microscopy of Aspergillus fumigatus keratitis. Br J Ophthalmol 2003;87:409-410.

14 Hau SC, Dart JKG, Vesaluoma M, et al: Diagnostic accuracy of microbial keratitis with in vivo confocal microscopy. Br J Ophthalmol 2010;94:982-987.

15 Labbé A, Gabison E, Cochereau I, et al: Diagnosis of fungal keratitis by in vivo confocal microscopy: a case report. Eye (Lond) 2011;25:956-958.

16 Vengayil S, Panda A, Satpathy G, et al: Polymerase chain reaction-guided diagnosis of mycotic keratitis: a prospective evaluation of its efficacy and limitations. Invest Ophthalmol Vis Sci 2009;50:152-156.

17 Tananuvat N, Salakthuantee K, Vanittanakom N, et al: Prospective comparison between conventional microbial work-up vs PCR in the diagnosis of fungal keratitis. Eye (Lond) 2012;26:1337-1343. 
Case Reports in

Ophthalmology

\begin{tabular}{l|l}
\hline Case Rep Ophthalmol 2013;4:303-310 \\
\hline DOI: $10.1159 / 000357558$ & $\begin{array}{l}\text { C 2013 S. Karger AG, Basel } \\
\text { www.karger.com/cop }\end{array}$ \\
\hline
\end{tabular}

Nielsen et al.: Fungal Keratitis - Improving Diagnostics by Confocal Microscopy

Table 1. Results

\begin{tabular}{|c|c|c|c|c|c|c|c|c|c|}
\hline Patient & $\begin{array}{l}\text { Age, } \\
\text { years }\end{array}$ & Fungus & Risk factors & $\begin{array}{l}\text { IVCM-positive } \\
\text { scans/N }\end{array}$ & $\begin{array}{l}\text { Culture-positive } \\
\text { cultures/N }\end{array}$ & Histopathology & $\begin{array}{l}\text { Delay, } \\
\text { days }\end{array}$ & Treatment type & $\begin{array}{l}\text { BSCVA, Snellen } \\
\text { decimal units }\end{array}$ \\
\hline 1 & 12 & F. solani & contact lens & $1 / 1$ & $1 / 3$ & negative & 45 & $\begin{array}{l}\text { amphorericin } \mathrm{B} \text {, } \\
\text { voriconazole, } \mathrm{PK}\end{array}$ & 0.63 \\
\hline 2 & 48 & Fusarium & $\begin{array}{l}\text { contact lens, } \\
\text { infected in } \\
\text { Thailand }\end{array}$ & $1 / 1$ & $0 / 2$ & positive & 40 & $\begin{array}{l}\text { amphorericin } \mathrm{B} \text {, } \\
\text { voriconazole, } \mathrm{PK} \text {, } \\
\text { enucleation }\end{array}$ & $-\mathrm{L}$ \\
\hline 3 & 55 & $\begin{array}{l}\text { Filamentous } \\
\text { fungus }\end{array}$ & $\begin{array}{l}\text { contact lens, } \\
\text { Pseudomonas } \\
\text { coinfection }\end{array}$ & $1 / 1$ & $1 / 1$ & not performed & 32 & $\begin{array}{l}\text { amphorericin B, } \\
\text { voriconazole }\end{array}$ & 0.8 \\
\hline 4 & 58 & F. solani & contact lens & $1 / 1$ & $1 / 4$ & positive & 55 & $\begin{array}{l}\text { amphorericin } \mathrm{B}, \\
\text { voriconazole, } \mathrm{PK} \times 2\end{array}$ & HM \\
\hline 5 & 25 & A. fumigatus & contact lens & $1 / 2$ & $1 / 4$ & not performed & 46 & $\begin{array}{l}\text { amphorericin B, } \\
\text { voriconazole }\end{array}$ & 0.8 \\
\hline 6 & 69 & F. solani & contact lens & $1 / 1$ & $2 / 3$ & not performed & 28 & $\begin{array}{l}\text { amphorericin } B \text {, } \\
\text { voriconazole }\end{array}$ & $\mathrm{HM}^{\mathrm{a}}$ \\
\hline Mean/total & 44.5 & & & $6 / 7$ (86\%) & $6 / 17(35 \%)$ & & 41 & & \\
\hline
\end{tabular}

BSCVA = Best spectacle-corrected visual acuity; $\mathrm{HM}=$ hand movements; $-\mathrm{L}=$ no light perception; $\mathrm{PK}=$ penetrating keratoplasty . a Still receiving treatment. 


\section{Case Reports in \\ Ophthalmology}

\begin{tabular}{l|l}
\hline Case Rep Ophthalmol 2013;4:303-310 \\
\hline DOI: 10.1159/000357558 & $\begin{array}{l}\text { @ 2013 S. Karger AG, Basel } \\
\text { www.karger.com/cop }\end{array}$ \\
\hline
\end{tabular}

Nielsen et al.: Fungal Keratitis - Improving Diagnostics by Confocal Microscopy
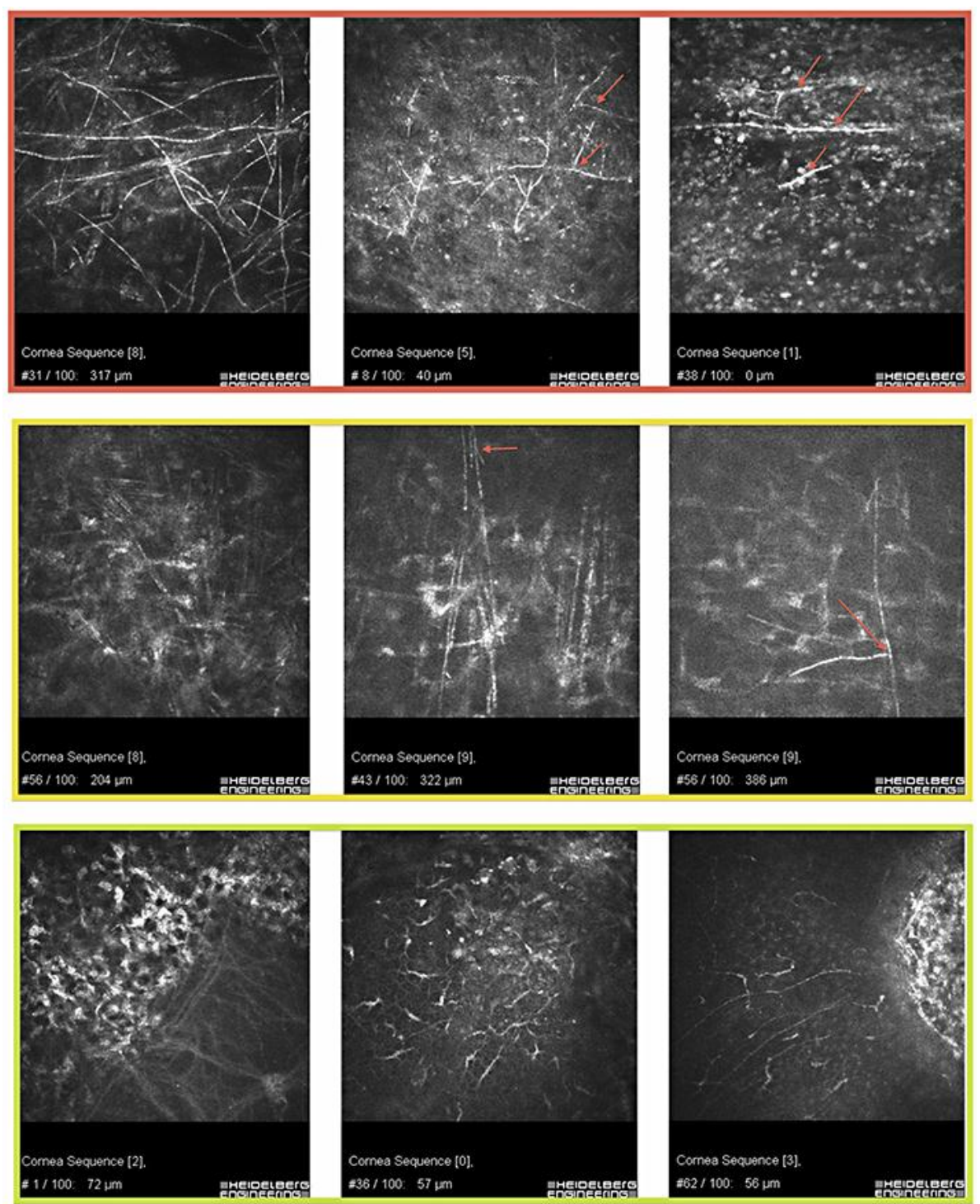

Fig. 1. Image grading in $F$. solani keratitis. Top row: dense stromal $F$. solani hyphae of slightly different thicknesses (left panel) and $F$. solani hyphae that appear as isolated branch-like elements (red arrows) in superficial layers. Middle row: stromal elements that could be hyphae but lack the interconnecting structures - this patient had bacterial keratitis (left panel). Stromal atypical elements that could represent nerves but because they bifurcate (red arrows); there is an indication that they represent hyphae - these patients had F. solani keratitis. Bottom row: an oblique section through Bowman's membrane in a cornea with Fuchs' endothelial dystrophy; there is some subepithelial fibrosis (left panel). Dendritic cells in a normal cornea (middle panel). Subbasal nerves at the edge of an infiltrate - bacterial keratitis (right panel). 


\section{Case Reports in \\ Ophthalmology}

\begin{tabular}{l|l}
\hline Case Rep Ophthalmol 2013;4:303-310 \\
\hline DOI: 10.1159/000357558 & $\begin{array}{l}\text { @ 2013 S. Karger AG, Basel } \\
\text { www.karger.com/cop }\end{array}$ \\
\hline
\end{tabular}

Nielsen et al.: Fungal Keratitis - Improving Diagnostics by Confocal Microscopy
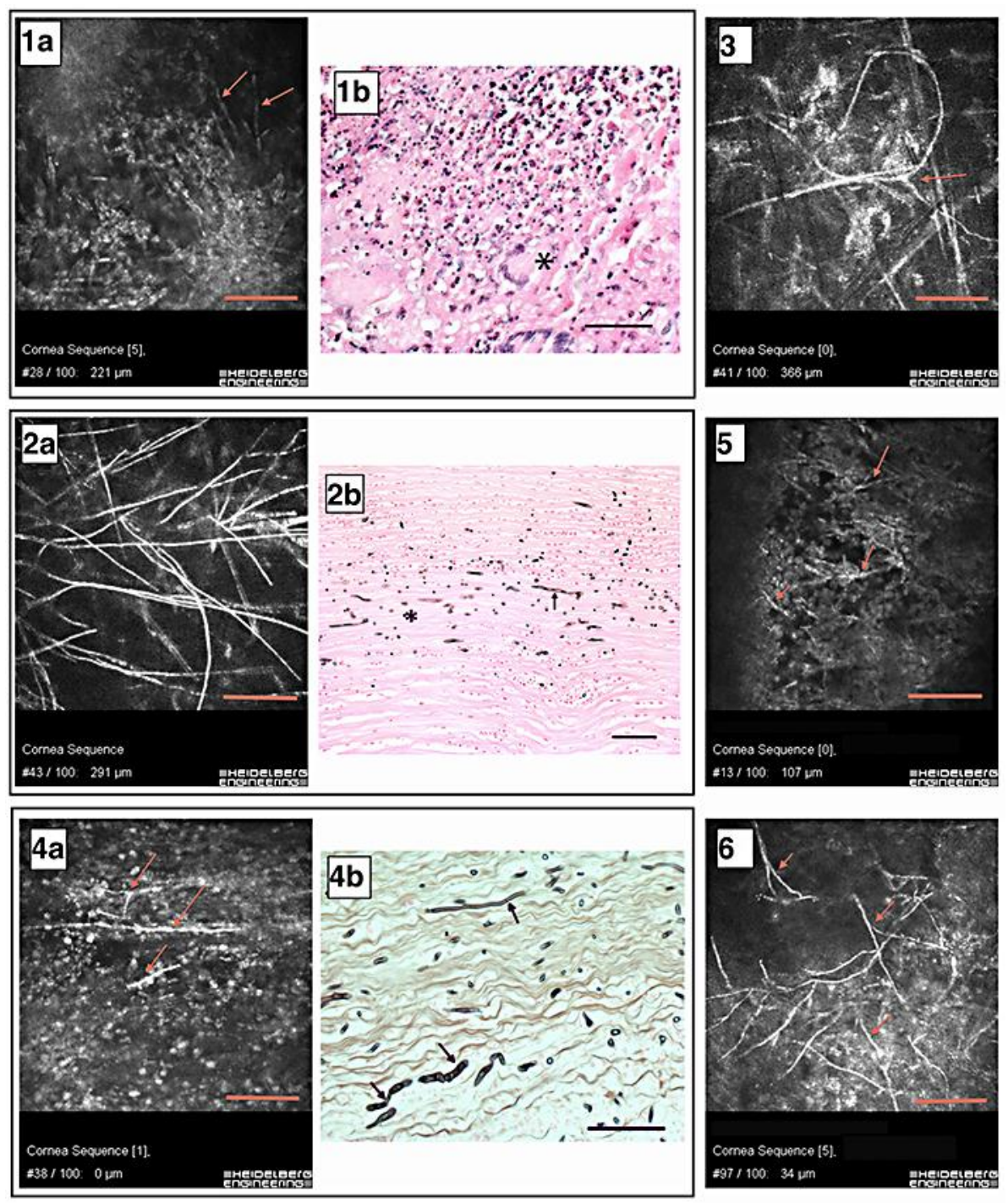

Fig. 2. IVCM results and histopathological images. Numbers correspond to patient numbers in table 1 . Scale bars $=100 \mu \mathrm{m}$. 1a Hyphae in the corneal stroma (red arrows). 1b Corneal button with severe granulomatous inflammation. Asterisk: foreign body giant cell. HE. 2a A stromal meshwork of hyphae. 2b Cornea with septated hyphae (arrow) and cross-sectioned hyphae (asterisk). Grocott. 3 Interlocking and irregularly shaped hyphae with a bifurcature (red arrow). 4a Red arrows show isolated hyphae within the epithelium. 4b Cornea with hyphae of various dimensions (arrows). Grocott. 5 Red arrows show thick bifurcating hyphae. 6 Red arrows show epithelial hyphae. 